\title{
Innovations in Information Retrieval: Perspectives for Theory and Practice
}

\author{
Allen Foster and Pauline Rafferty, Facet Publishing, London, $£ 42.70$ (paperback)
}

\author{
George Macgregor \\ Information Services Directorate \\ University of Strathclyde, Glasgow, UK
}

Often the most difficult process for a postgraduate research student is not the process of research; many may find the research process arduous and bewildering but - with adequate supervision and a robust research plan - students tend to cope well with the methodological demands and data collection. Instead, where students struggle is identifying a topic worth studying in the first place (Millman, 1998). This scenario is not limited to the information, computer, or library sciences. It is a phenomenon that often transcends disciplinary boundaries and academic levels of study (Hirt and Muffo, 1998). Students may have the faint inkling of a topic area but may have insufficiently assimilated extant literature, or simply struggled to understand the research landscape, and in so doing failed to articulate a satisfactory research topic with any degree of specificity.

Allen Foster and Pauline Rafferty are both lecturers at the University of Aberystwyth and are presumably familiar with the aforementioned phenomenon through their professional practice because it is precisely this that Innovations in Information Retrieval seeks to resolve. Its raison d'être is to "inspire Master's-level students who might be looking to develop their dissertation topics [in information retrieval (IR)], or indeed to develop PhD proposals" (p. xv). To this end Foster and Rafferty present seven chapters, each focussed on a specific aspect of IR and each authored by experts in the field.

David Bawden (City University, London) provides the opening chapter in which he considers the continuing relevance of browsing and serendipity in IR. The evolution of information-seeking behaviour theory is examined through the prism of prevailing IR trends, resulting in Bawden's proposal that there should be greater exploration of "serendipity systems" which are capable of better supporting creativity and innovation in the user. Aida Slavic (UDC Editor-in-Chief and Visiting Lecturer at University of Zagreb) continues the browsing metaphor in chapter 2 with her revisitation of knowledge organisation systems. Slavic manages to condense the essence of taxonomic classifications and features such facet analytical theory, whilst simultaneously highlighting current research trends in IR, automatic classification, terminology services, the semantic web and linked data.

Chapters 3 and 4 move the book into niche areas of IR research: fiction IR and music information retrieval (MIR). Anat Vernitski (UK Data Archive) and Pauline Rafferty take the reader on an exploration of the issues pervading literary IR, including an overview of Vernitski's prototype fiction retrieval system and proposals for a generic fiction retrieval system. Similarly, Charlie Inskip (London Metropolitan University) ably reviews the research landscape of MIR and, like the chapter before it, articulates the peculiar issues that pervade multimedia retrieval. Of course, these peculiarities are what drives the research agenda and Inskip summarises specific issues pertaining to MIR evaluation, the impact of social media, and mood and emotion analysis as being worthy of further investigation.

Perhaps one of the most significant developments in IR over recent years has been social media and the emergence of "social search". Almost all the chapters in Innovations [...] mention it, at least in 
passing; however, Isabella Peters' (Heinrich Heine University, Düsseldorf) chapter focuses almost exclusively on this area, in particular on the extent to which social tagging meets the demands of social searching. Peters provides a comprehensive snapshot of associated research in areas such as recommender systems, tag clouds, and relevance ranking, and concludes that the most productive areas for future research are visualisation techniques, search engine design, results ranking, and greater investigation of how user activity can best be harnessed for IR. Peters' focus on knowledge representation segues nicely into a contribution from Richard Kopak, Luanne Freund and Heather O'Brien (all based at University of British Columbia). Their chapter on semantic navigation is perhaps the most useful for the research hungry student, dividing as it does its content into distinct sections, some of which are headed "Areas for further research: annotation", "Areas for future research: linking", and so forth. Kopak et al. outline the need for IR systems to better support the information interaction habits of modern users. Such support can only come from improved use of semantic navigation so that as users "move through information they actively construct meaning and make use of both explicit and implicit features of the information and its environment" (p. 118). And of the research areas delineated within the chapters of Innovations in Information Retrieval, Kopak et al.'s is arguably the most embryonic, providing students with a fertile field of potential research avenues. Webometrics expert, Mike Thelwall (University of Wolverhampton) concludes the book by reviewing research on webometric analyses of web search engines and, like Kopak et al., he provides a veritable shopping list of interesting issues for students to explore.

In its quest to present "hot" research topics Innovations in Information Retrieval certainly succeeds. Each chapter provides an exhaustive snapshot of its research area and summarises the literature admirably, providing a comprehensive list of seminal literature that research students would enjoy chaining. One possible criticism, however, is the editorial scope of the book. A seven chapter volume is insufficient to capture the multitude of exciting and thriving IR research areas. The editors by no means purport Innovations [...] to be definitive; but since a book of 14 chapters is unlikely, it is worth pondering whether the contributions selected for seven chapters could have been a little more diverse. For example, there is some minor intellectual overlap between chapters and one wonders whether some could have been sacrificed for a chapter on IR in, say, personal information management contexts, or topics within interactive IR. This, however, is a minor criticism of what is otherwise a useful text for any student considering research within the general area of IR. In some ways Innovations [...] may have better suited a special issue of Aslib Proceedings or some such. No doubt this thought occurred to the editors but was eschewed for fear that it might exclude the intended readership. But it is all swings and roundabouts; the price tag of Innovations in Information Retrieval might, unfortunately, deter some students from making the investment.

\section{References}

Hirt, J.B. and Muffo, J.A. (1998), "Graduate students: institutional climates and disciplinary cultures", New Directions for Institutional Research, Vol. 1998 No. 98, pp. 17-33.

Millman, J. (1998), "Strategies for identifying research topic in educational measurement", Educational Measurement: Issues and Practice, Vol. 17 No. 2, pp. 37-9 